\section{A brief parent-focused intervention reduces anxiety disorders in socially inhibited children}

\section{QUESTION}

Question: Can future anxiety disorders be prevented in inhibited preschool-age children following a parent-focused intervention?

Patients: 146 preschool-age children and their parents. Children were aged on average 3 years 10 months and were initially reported by their mothers to have a high level of withdrawal in prestudy screening questionnaires (the approach subscale of the Short Temperament Scale for Children). They were subsequently included if they demonstrated behavioural inhibition in further laboratory assessment.

Setting: Recruitment between 1998 and June 2000 from Australian preschools.

Intervention: Parents were randomised to receive an intervention or monitoring only. The parent-intervention programme included small groups of parents invited for six 90 min sessions with a clinical psychologist. The first four sessions were held weekly, the fifth was 2 weeks later and the final was held a month later. The sessions included discussions about the nature of anxiety and its development, parent management techniques focusing on how overprotection maintains anxiety, sessions teaching the application of cognitive restructuring to the parents own worries and important high-risk periods for children such as starting school. Parents in the monitoring-only group did not receive any intervention and were contacted at each follow-up period.

Outcomes: Diagnosis of anxiety disorders and anxiety symptoms. Self-assessments were repeated at 12, 24 and 36 months, whereas laboratory observations were repeated at 12 and 24 months. The final assessment occurred when the children were around 7 years old.

Patient follow-up: $83 \%$ of the parents who were randomised provided two or more data points for analysis.

\section{METHODS}

Design: Randomised controlled trial.

Allocation: Not clear.

Blinding: Non-blinded.

Follow-up period: Around 2-4 years.

\section{MAIN RESULTS}

Children whose parents received the intervention showed lower frequency and severity of anxiety disorders. The average anxiety diagnoses in both groups reduced between baseline and 36 months, but this was numerically greater in the intervention group (change in mean: 1.49 with intervention vs 0.81 with monitoring only; significance not reported). The severity of anxiety also reduced between baseline and final follow-up in both groups, but this was greater in the intervention group (change in severity score on clinician rated 8-point scale: 3.02 with intervention vs 0.91 with monitoring only; significance not reported). In mixed model analysis, the group-by-time interaction was significant, indicating a significant effect of treatment on change in mean number of anxiety diagnoses from baseline ( $p=0.032$ from baseline to 36 months). The group-by-time interaction was also significant for the average clinical severity of anxiety symptoms $(\mathrm{p}=0.002)$.

\section{CONCLUSIONS}

A parent-focused intervention for parents of socially inhibited preschool children reduces subsequent anxiety disorder diagnoses in children. The component of the intervention responsible for this effect was not determined. The intervention did not, however, improve inhibition in children, a key risk factor for subsequent anxiety disorders.

\section{ABSTRACTED FROM}

Rapee RM, Kennedy SJ, Ingram M, et al. Altering the trajectory of anxiety in at-risk young children. Am J Psychiatry 2010;167:1518-25.

Correspondence to: Dr Rapee, Department of Psychology, Macquarie University, Sydney, NSW 2109, Australia; ron.rapee@mq.edu.au

Sources of funding: National Health and Medical Research Council.
A nxiety problems are relatively common in children, and, while evidence is strong that tertiary and preventive interventions are effective in late childhood onwards, little is available to suggest we can successfully prevent anxiety problems earlier in childhood. Thus, the randomised controlled study by Rapee and colleagues is of considerable interest.

Can we prevent anxiety disorders by targeting early childhood? This study is a great start, and the authors are to be congratulated for succeeding with such a difficult study. I remain cautious for several reasons. First, the participants were largely affluent volunteers from middle class to prestigious areas of Sydney ( $>50 \%$ of parents had a university education). Second, the putative mechanism of change - behavioural inhibition - did not change, and thus it is unclear which components of the intervention produced the effects; I worry about reporting biases from parents who committed to the intervention group. Third, most anxiety disorders emerge in late childhood/adolescence, and so it is unclear how the intervention will fare through the important developmental age these children are yet to traverse. As Cuthbert ${ }^{1}$ observed, differences between the intervention and control groups emerged over time and may be due to a sharp increase in symptoms for the control group. This might indicate that the cohort is entering the high-risk period and that the intervention is actually preventing onset for the children who received it. The next few years will be critical for testing this.

These results are tantalising; as a prevention scientist/clinician, however, I would caution about moving those rare prevention resources to earlier ages just yet. There is a strong evidence base for successful prevention of anxiety problems in late childhood/ early adolescence. Previous studies have struggled to find prevention effects in early childhood, ${ }^{2-4}$ so further studies using more representative parents are needed to replicate these effects and show that they are durable through the risky adolescent period.

\section{Mark R Dadds}

School of Psychology, The University of New South Wales, Sydney, New South Wales, Australia

Competing interests None.

\section{REFERENCES}

1. Cuthbert B. Early prevention in childhood anxiety disorders. Am J Psychiatry 2010;167:1428-30.

2. Rapee RM, Kennedy S, Ingram M, et al. Prevention and early intervention of anxiety disorders in inhibited preschool children. J Consult Clin Psychol 2005:73:488-97.

3. LaFreniere PJ, Capuano F. Preventive intervention as means of clarifying direction of effects in socialization: anxious-withdrawn preschoolers case. Dev Psychopathol 1997; 9:551-64.

4. Dadds MR, Roth JH. A universal intervention trial for preventing anxiety problems in young children. J Child Family Studies 2008;17:320-35. 